\title{
Marie-Claude Blais, Marcel Gauchet, Dominique Ottavi, Conditions de l'éducation
}

Stock, 2008, 265 p.

Anne-Marie Bardi

\section{OpenEdition}

\section{Journals}

Édition électronique

URL : http://journals.openedition.org/ries/629

DOI : $10.4000 /$ ries.629

ISSN : 2261-4265

Éditeur

Centre international d'études pédagogiques

Édition imprimée

Date de publication : 1 septembre 2009

Pagination : 23-25

ISSN : $1254-4590$

Référence électronique

Anne-Marie Bardi, « Marie-Claude Blais, Marcel Gauchet, Dominique Ottavi, Conditions de l'éducation », Revue internationale d'éducation de Sèvres [En ligne], 51 | septembre 2009, mis en ligne le 01 septembre 2011, consulté le 22 septembre 2020. URL : http://journals.openedition.org/ries/629 ; DOI : https:// doi.org/10.4000/ries.629

Ce document a été généré automatiquement le 22 septembre 2020.

(c) Tous droits réservés 


\section{Marie-Claude Blais, Marcel Gauchet, Dominique Ottavi, Conditions de l'éducation}

Stock, 2008, 265 p.

Anne-Marie Bardi

\section{RÉFÉRENCE}

Marie-Claude Blais, Marcel Gauchet, Dominique Ottavi, Conditions de l'éducation,

Stock, 2008, $265 \mathrm{p}$.

1 L'École n'est pas en très bonne santé. Beaucoup le déplorent et tentent, à leur niveau de responsabilité, de proposer des remèdes. En France, le gouvernement a récemment légiféré, l'administration centrale de l'éducation nationale poursuit son processus de déconcentration, les responsables territoriaux - recteurs et inspecteurs d'académie modernisent leur mode de pilotage, les inspecteurs et chefs d'établissement privilégient l'accompagnement des acteurs plutôt que leur encadrement strict et les enseignants ne cessent d'innover et de coopérer pour améliorer leurs pratiques. Sans résultats tangibles... Nombre des maux dont souffre l'école seraient-ils dus non à sa propre constitution - certes complexe voire fragile - mais à son environnement ? C'est la piste proposée par les auteurs: l'évolution de nos sociétés ne remet-elle pas en question les conditions mêmes de "possibilité de l'entreprise éducative»? L'investigation proposée au lecteur se développe dans quatre directions: la relation famille/école, le sens des savoirs, la fonction de l'autorité et l'articulation société/école. Les auteurs considèrent que chacun de ces domaines contribuait par le passé au projet éducatif global et qu'ensemble ils constituaient le socle de l'institution éducative. Des transformations majeures de la société ont ébranlé ce socle; il faut commencer par mettre en lumière les effets de ces transformations pour comprendre les difficultés sur lesquelles l'éducation bute. 
2 La première investigation concerne la famille; c'est en effet l'espace premier, celui où l'enfant est conçu, accueilli, et élevé. Son rôle est ici traité dans une perspective historique : la famille « traditionnelle » se devait d'inscrire l'enfant dans un cadre quasi immuable - agriculture, artisanat, commerce - et se satisfaisait d'assurer elle-même une éducation fondée sur la conformité à un ordre stable et à des hiérarchies établies ; la famille moderne, dont l'apogée se situerait à la fin du XIX ${ }^{e}$ siècle, s'est ensuite constituée en articulation étroite avec le monde économique et politique. L'enfant étant appelé à vivre dans un monde plus vaste et à exercer des métiers divers, l'institution scolaire, à vocation universelle, complétait naturellement l'éducation familiale. L'intérêt de la collectivité - donc l'organisation de l'École - était en plein accord avec les aspirations légitimes des parents.

3 Aujourd'hui, la visée des familles ne semble plus correspondre aux finalités de l'éducation. Si les parents demeurent soucieux du devenir de leurs enfants, ils souhaitent paradoxalement les préserver de la pression scolaire et rêvent d'une école sereine et accueillante adaptée à chaque enfant dans sa singularité. L'auteur conduit une étude précise des modifications récentes de la structure familiale et de leurs conséquences sur la place de l'enfant et sur son éducation. Elle se réfère par exemple à une étude menée en 2003 en Amérique montrant que, "si tous les parents sont intéressés au bonheur de leurs enfants, peu d'entre eux conçoivent celui-ci dans son lien avec une insertion dans une société traversée par des règles et des codes ». S'ensuit souvent un véritable déni des institutions sociales, ce qui ne permet pas à l'enfant de comprendre sa dépendance vis-à-vis du passé ni de se projeter dans la vie de la collectivité et lui fait ressentir les normes et les règles indispensables aux apprentissages comme arbitraires, voire absurdes. Le titre de cette partie en indique bien l'esprit : « La famille, contre l'éducation? ».

4 Second pilier de toute éducation, la valeur intrinsèque des savoirs et l'évidence de la nécessité de les acquérir. Aujourd'hui, il est banal de constater que les élèves s'ennuient à l'école. Justifier les contenus enseignés, les modifier ou changer les méthodes pédagogiques n'y a rien changé. L'auteur constate que les savoirs semblent privés de sens et il en présente les causes : le rapport au passé, le mode de socialisation, enfin le statut social de la connaissance, du savoir et de la culture.

5 La « détraditionalisation » implique la patrimonialisation : le passé est mort et muet, on le visite, on le respecte, mais il ne nous est plus indispensable. Au temps où le passé était reconnu comme exemplaire, l'École, avec une ambiguïté assumée, développait des méthodes modernes, rejetait les modes traditionnels de transmission mais, dans le même temps, tenait sa légitimité de sa qualité de truchement avec une antériorité modèle : « l'évidence inquestionnée d'une obligation envers le passé » a disparu. Autre conséquence, l'individualisme prime désormais sur le sentiment d'appartenance sociale et d'inscription personnelle dans un collectif. Le besoin de société s'effaçant, ce sont les besoins, les désirs et les intérêts de chacun qui comptent désormais. Dernier ensemble de causes, l'extériorisation des savoirs. Avec soin, l'auteur distingue savoirs, connaissances et culture et décrit les profondes ruptures apparues aujourd'hui par rapport à leurs fonctions antérieures. Impossible de rendre compte, en quelques lignes, de cette démonstration brillante, reprenons la conclusion: "Que faire de savoirs qui "prennent la tête" dans un monde où l'aspiration primordiale est à être "bien dans sa peau" ? ». Deux annexes complètent l'analyse globale des causes de la perte du sens des savoirs, l'une pour le domaine littéraire, l'autre pour le domaine scientifique. 
6 La troisième partie du livre s'intitule «Fin ou métamorphose de l'autorité ? ». Elle interroge, au plan théorique, la nature de l'autorité, soigneusement distinguée des notions de pouvoir et de puissance. Des considérations historiques et philosophiques en éclairent les différentes facettes: le fait de l'autorité, les raisons, les expressions et enfin les fonctions de l'autorité. Dans le champ éducatif, l'autorité constitue la seule voie qui puisse concilier les termes antinomiques de l'expression «former des individus ", entre la nécessité de passer par les autres pour s'instruire et le respect de l'indépendance de l'individu qui se construit. Mais cette autorité n'a rien de naturel et elle s'est peu à peu déconstruite avec l'effacement de la légitimité de l'institution. La reconstruire sur des bases nouvelles autour d'un consensus rationnel des citoyens est impératif pour que soit rendu possible tout projet éducatif.

Dernier axe, celui de la vie de l'enfant, de son quotidien, de la société qu'il perçoit ; un quotidien mal connu, rempli d'activités diverses complémentaires de l'école, de télévision et d'ordinateurs. Un « enfant-roi » ou un enfant coupé de « la vraie vie »? Un enfant ignorant du monde du travail, échangeant peu avec les adultes - d'où une maitrise de la langue parfois peu assurée -, manquant souvent de repères, voire de modèles, en butte aux violences des médias et, parfois, aux pressions sociales de ses pairs. Ignorant de la société, vivant dans un monde cloisonné, l'élève peine à comprendre à quoi au juste le prépare l'école.

8 L'ouvrage suscite et stimule la réflexion. Chacun des trois auteurs traite un ou plusieurs thèmes avec son propre style, ce qui permet de gagner en pertinence et en clarté mais n'évite pas quelques répétitions. La force de l'ouvrage tient à l'originalité et à la pertinence de son approche, à l'érudition de ses auteurs et à la qualité de leur écriture. Mais elle réside aussi, et peut-être d'abord, dans le projet lui-même. Loin de démobiliser un lecteur qui verrait à l'œuvre des évolutions inéluctables, loin de promouvoir la nostalgie d'un âge d'or révolu, les auteurs nous livrent les clés d'une réflexion neuve. Poser les problèmes, comprendre en profondeur les tensions actuelles c'est «prendre la mesure de la tâche de refondation qui est devant nous ». Le livre y engage et fournit à chacun de nouvelles armes pour affronter les difficultés, qu'il soit acteur de l'éducation ou simple citoyen.

\section{AUTEURS}

\section{ANNE-MARIE BARDI}

Anne-Marie Bardi est Inspectrice générale (H) de l'Éducation nationale (mathématiques). Elle a été professeur puis Inspectrice d'académie-Inspectrice pédagogique régionale de mathématiques et s'est impliquée dans le développement de l'usage de l'informatique puis des technologies de l'information et de la communication dans l'enseignement. Elle est membre du comité de rédaction de la Revue internationale d'éducation de Sèvres. 